\title{
Formation of a Closing Apparatus of the Lower Uterine Segment in Patients with Invasive Cervical Cancer after Radical Transabdominal Trachelectomy
}

*Corresponding author:

A.L. Chernyshova,

email: alacher@list.ru

Received: 23 March 2017

Accepted: 9 April 2017

Published: 16 July 2017

\section{Publishing services}

provided by Knowledge E

Copyright (C 2017 A.L. Chernyshova et al. This article is distributed under the terms of the Creative Commons Attribution License, which permits unrestricted use and redistribution provided that the original author and source are credited.

Selection and Peer-review under the responsibility of the SMBIM Conference Committee.

\section{A.L. Chernyshova ${ }^{1 *}$, L.A. Kolomiets ${ }^{1}$, E.S. Marchenko ${ }^{2,3}$, G.A. Baigonakova ${ }^{2,3}$, V.E. Gyunter ${ }^{2,3}$}

${ }^{1}$ Oncology Research Institute of Tomsk Scientific Research and Marketing Centre, Tomsk, Russia

${ }^{2}$ Tomsk StateUniversity, Tomsk, Russia

${ }^{3}$ Research Institute of Medical Materials and Implants with Shape Memory, Tomsk, Russia

\section{Abstract}

Strengthening of the lower uterine segment and formation of a "closing apparatus" for carrying a subsequent pregnancy in the absence of the cervix in patients with cervical cancer after conserving treatment in the amount of radical transabdominaltrachelectomy. Materials and Research Methods: the study included 26 patients with cervical cancer of stage I in the reproductive age who received treatment in the amount of radical transabdominaltrachelectomy (RTT). In the course of surgery in the amount of RTT, after an imposition of an utero-vaginal anastomosis, the strengthening of the lower uterine segment by setting and fixing a mesh of TiNi and its modelling within the range from the lower uterine segment to the upper third of the vagina was performed. Results: with regard to the issue of strengthening the utero-vaginal anastomosis, the implant takes over the function of the basic "closing apparatus" of the uterine and provides functional support and stability of the utero-vaginal anastomosis against the effects of intrauterine and intraperitoneal pressure. Conclusion: the usefulness of a mesh implant of the superelasticTiNi is due to its biomechanical properties: it is not absorbed, but gets fused with the surrounding tissue and provides resistance of tissues to excessive strain.

\section{G OPEN ACCESS}




\section{Introduction}

Over the past 10 years in the Russian Federation up to 15 thousand first time cases of cervical cancer (CC) have been recorded annually, which is about $7 \%$ of the total number of cancer patients. Cervical cancer is most often diagnosed in women aged 40-60 years old, while in recent years there has been a negative trend - an increase in the incidence of women of the reproductive age with an annual growth rate of $2-7 \%$, a similar trend continuing in the Siberian region $[1,2]$. In this connection, new medical technologies of treating cervical cancer in women of the childbearing age are currently being actively developed, as evidenced by numerous publications in the world literature [3].

Nowadays, one of the priorities of modern oncology is conserving treatment $[4,5$, 6]. According to FIGO data, in CC patients with cervical tumour dissemination process of more than $3 \mathrm{~mm}$, the process is considered invasive, and in such cases the treatment is conducted by aggressive methods.

At early stages of cervical cancer (IB1 and IIA stages with the size of the tumour equal to less than $4 \mathrm{~cm}$ ) the prognosis is favourable $[7,8]$. In treatment of invasive cervical cancer, until recently, the classical methods were the surgical and the combined methods at all stages of the disease. Surgical treatment implies performing the Wertheim operation and, if combined treatment is necessary, applying radiation therapy. Naturally, holding these therapies is accompanied by the loss of fertility [9]. At present, the conservative operation of radical trachelectomy for treatment of patients with cervical cancer of IA2-IB2 stages is developed and widely implemented (FIGO) [10, 11].

The origins of organ-preserving treatment of invasive cervix cancer are commonly considered to date back to 1950s, when the Romanian surgeon E. Aburel was the first to publish an article in a Romanian magazine that expressed the idea of the possibility to conduct extended abdominal trachelectomy in cases of in situ cancer and microinvasive cervical cancer [12].

At the beginning of the XXI century gynaecological oncologists took an interest in extended trachelectomy performed by the transabdominal access again, and the undisputed leaders of this trend have been a group of authors from Hungary, the 
UK and the USA $[13,14]$. Performing the surgery in this way provides a more radical removal of the vaginal cuff, paracervical and paravaginal fibre, especially in the case of the uterine vessels ligation in the place of their discharge from the internal iliac vessels and, in fact, it is comparable to extended hysterectomy $[15,16]$. In addition, the implementation of expanded trachelectomy with an application of the abdominal access is also possible for larger tumours - even up to $6 \mathrm{~cm}$ at IA2-IB2 stages (FIGO) [17]. Carrying organ-preserving treatment in patients with cervical cancer of IB1, and in some cases, IV2 and IIA stages, (selection criteria - tumour size of less than $4 \mathrm{~cm}$, no signs of metastasis) is possible in the amount of radical trachelectomy [18]. The amount of tissue removed during the operation is similar to the Wertheim operation, but the body of the uterus and the appendages are preserved. Currently, the oncological effectiveness is proved and the data on a successful pregnancy and delivery after radical trachelectomy are available [19].

As for the histological tumour type, according to the results of the investigations, the incidence of cervical cancer progression as in squamous cell carcinoma and adenocarcinoma has no statistical differences [19].

Currently, various modifications of trachelectomy surgery are considered. In the first place, they are the imposition of a reanastomosis of the uterine arteries in order to improve the uterine blood supply and to improve the conditions for the implantation of the ovum, as well as the formation of the uterine-placental complex and the gestation period [5].

One of the important and unresolved issues is the need to strengthen the lower uterine segment and the formation of a "closing apparatus" for carrying a subsequent pregnancy in the absence of the cervix. The closing function failure of the uterus isthmus (isthmus-cervical incompetence) can lead to mechanical lowering and a prolapse of the fetal bladder, which creates conditions for an infection. Moreover, there is a possibility of the fetal bladder interference into the anastomosis area, which contributes to a further expansion and a growing threat of termination of pregnancy, as well as development of the utero-vaginal anastomosis dehiscence. Currently, at the initial stages of pregnancy in healthy women in the case of cervical incompetence the common method of imposing a cerclage on the cervix is applied. Naturally, this operation is unfeasible in any versions in the case of cervical hysterectomy. For the category of patients with cervical cancer after radical trachelectomy the cerclage in the lower uterine segment is usually applied in the period from 8th to 17th week of pregnancy. At 
the same time, the possibility of preventing a failure of the utero-vaginal anastomosis in the early and late postoperative period, as well as directly during the surgery is actively discussed. However, the known methods of imposing the cerclage in the lower uterine segment area using dacron, silk, nylon and chromic catgut as the surgical suture cannot be recognized as sufficiently effective.

Using any of the above mentioned materials in any case creates additional conditions for infection and therefore requires an antimicrobial therapy. It is also necessary to consider the possibility of adverse effects of drugs (antibacterial products) on the fetus. The most frequent complications resulting from surgical treatment of isthmus-cervical incompetence after trachelectomy with sutures include teething of the anastomosis tissue, occurrence of bedsores with further formation of fistulas, as well as transverse or circular gaps [7].

In the available literature there are no sources which would represent sufficiently effective options for strengthening the lower uterine segment in order to form the uterine closing apparatus directly during radical abdominal trachelectomy. The aim of our study is to provide a method of forming the uterine closing apparatus in patients with cervical cancer after radical abdominal trachelectomy.

\section{Materials and Methods}

The study included 26 patients with cervical cancer of stage I in the reproductive age who received treatment in the amount of radical transabdominaltrachelectomy in the department of gynaecological oncology at the Federal State Funded Institution "Cancer Research Institute," from 2012 to 2014. Patients with cervical cancer of stage I were divided into the following sub-stages: la1 stage - 7 patients (27\%); la2 - 8 (31\%); Ib1- 11 patients (42\%). In all the cases non-keratinizing squamous cell carcinoma of different differentiation degrees was verified morphologically. The average age of the patients was $28.7 \pm 4.5$ years old.

During the surgical treatment in the amount of radical abdominal trachelectomy, after an imposition of the utero-vaginal anastomosis, the lower uterine segment was strengthened by a mesh of a superelasticTiNi thread (Fig. 1a, 1b). The installation and fixation of the TiNi mesh was carried out with modelling it within the area between the lower uterine segment and the upper third of the vagina (Fig. 2a, 2b). The fixation of the mesh was performed by putting four separate stitches along the perimeter. 

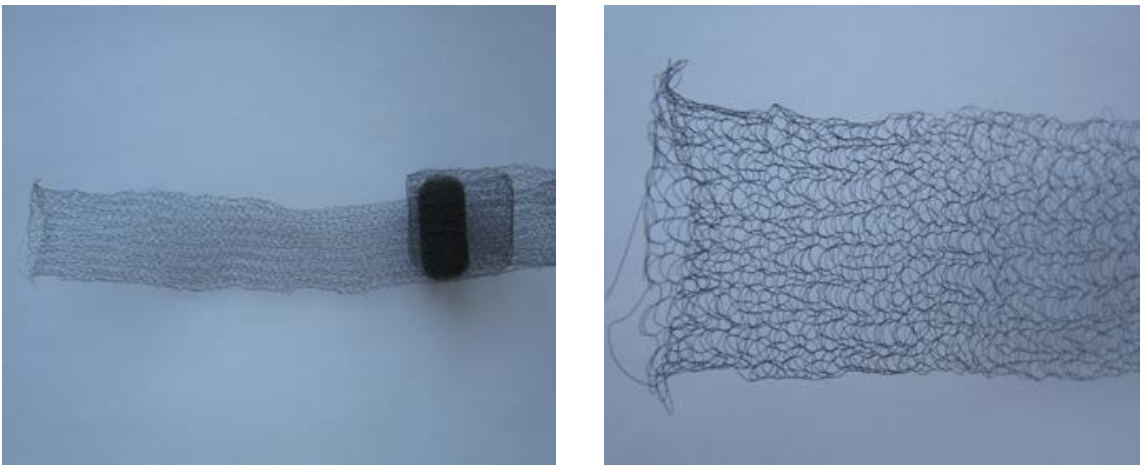

Fig. 1. SuperelasticTiNi mesh implant

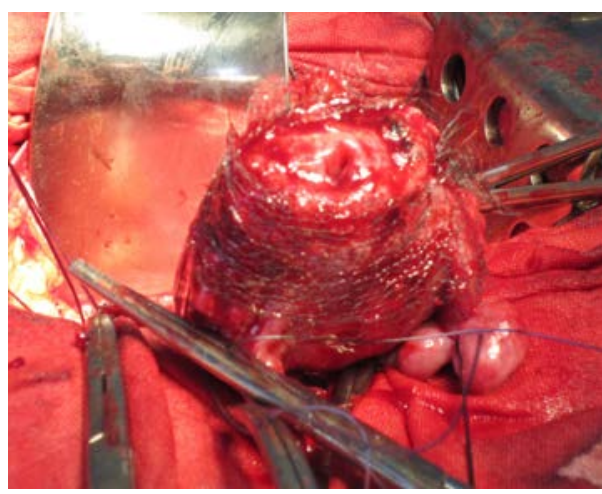

a

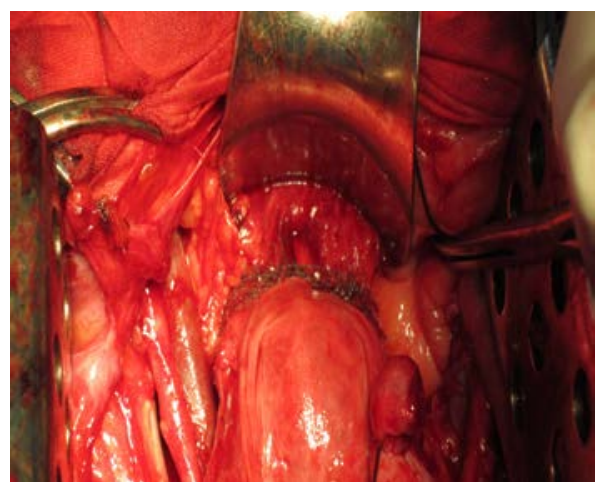

b

Fig. 2. Surgical wound type: a - fixation of mesh implant around lower uterine segment, $b$ - final form of surgical wound

The dimensions of the TiNi mesh (TN-1) were as follows: the width of $4-5 \mathrm{~cm}$; the length of $7-8 \mathrm{~cm}$; the thread diameter of $0.1-0.08 \mathrm{~mm}$; the mesh unit size of $3-5 \mathrm{~mm}$. The length of the mesh implant was in the range of $5-7 \mathrm{~cm}$, which corresponds to the anatomy of the defect and allows making at least one complete wrap around the anastomosis with a slight overlap.

The stages of the operation are shown in Fig. 2. After the removal of the distal portion of the cervix and the upper third of the vagina, in accordance with Fig. 2, the anastomosis between them is formed by means of suturing with a continuous thread along the perimeter which is further wrapped with the mesh implant (Fig. 3). The mesh implant has the form of a stocking with the width of $4-5 \mathrm{~cm}$ and is made of a superelasticTiNi tread with the diameter of $0.08-0.1 \mathrm{~mm}$ and the mesh size of $3-5 \mathrm{~mm}$. The implant length is of $7-8 \mathrm{~cm}$. The fixation of the implant for the purpose of primary immobilization is performed by putting four separate stitches along the perimeter (Fig. 3). 


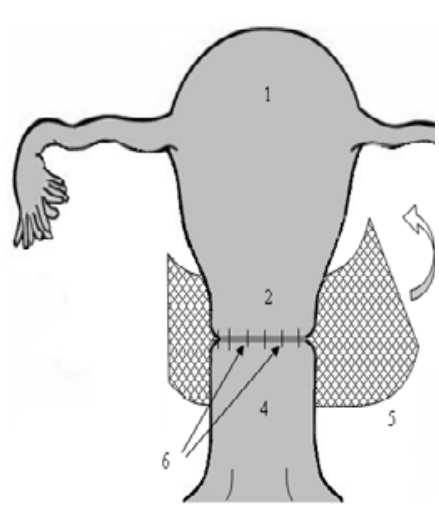

a

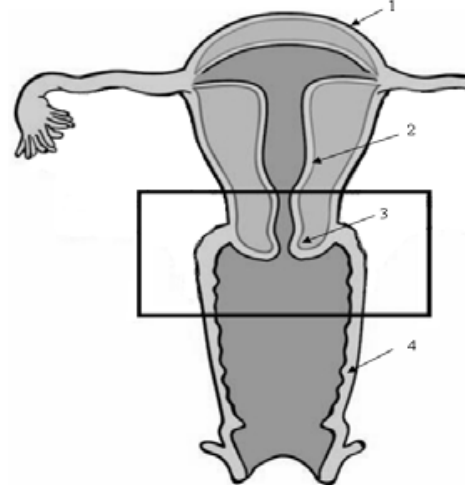

b

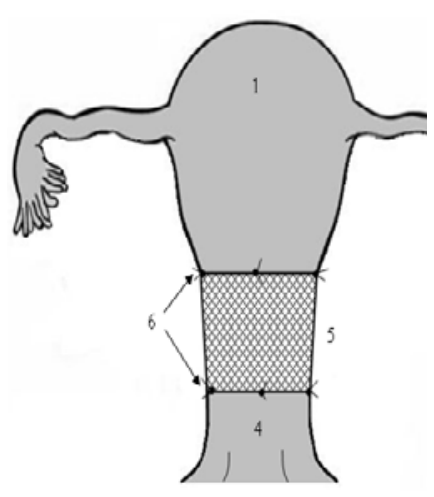

C

Fig. 3. a - Schematically represented operating zone area for case of conserving therapy of invasive cervical cancer;

b - schematically represented zone of implant installation and fixation;

c - schematically represented operating wound after implant fixation

The numbers refer to: 1 - the body of the uterus; 2 - the cervical canal; 3 - the cervix; 4 - the vagina; 5 - the mesh implant; 6 - the stitches.

The proposed method has been developed and tested in the gynaecology department of the Federal State Funded Institution "Institute of Oncology" of SB RAS (the positive decision on granting a patent from 5.03.2014).

The case follow-up of this group of patients in the postoperative period was performed using ultrasound monitoring of pelvic organs. In addition, in the postoperative period the conditions of the lower uterine segment and the area of the anastomosis were assessed by overview radiography.

Clinical example

The patient Z., 34 years old, was hospitalized in the Department of Gynaecology at the State Institution "Institute of Oncology" of SB RAS in February 2012. On admission, she complained of periodic aching drawing pains in the lower abdomen and bloody spotting from the genital tracts.

With this complaint, the patient consulted the gynaecological department of an antenatal clinic and was further appointed for hospitalisation at the Oncology Research Institute with the post-test diagnosis of suspected cancer of the cervix.

At the admission a complete clinical and laboratory examination was held. 
The examination revealed the following deviances: the cervix increased to $5 \mathrm{~cm}$ with signs of erosion and contact bleeding; scarce bloody discharge from the external os. The colposcopy results were as follows: the vaginal portion of the cervix covered by stratified squamous epithelium; in the area of the front lip of the uterine mouth and on the right external uterine os several sites with coarse mosaics on acetic whitish epithelium, as well as numerous atypical vessels in the form of coarse points; an increased vulnerability of the epithelium; within the range from 3 to $5 \mathrm{o}^{\prime}$ clock on the contingent dial elevated areas of an intense acetic-white colour, small bleeding erosions and atypical vascularization in the form of randomly arranged vessels of bizarre shapes which do not anastomise together (broken tangled blood vessels, capillary needle capillaries); around the external os atypical vessels in the form of points and an increased vessel vulnerability.

After applying Schiller samples in the area of the anterior and posterior lips of the uterine mouth numerous areas of epithelium with a negative reaction to iodine were visualized.

The impression smears for cytology were taken with a cervix-brush. The cytological conclusion number 2141 was as follows: non-squamous cervical cancer with symptoms of koilocytosis.

A biopsy of the cervix by colposcopy control was performed (section from 3 to 6 o'clock). The histological conclusion № 23001-44 was as follows: non-squamous cervical cancer of a moderate differentiation degree with an invasion up to $5 \mathrm{~mm}$, a spread up to $5-6 \mathrm{~mm}$ and cancerous emboli in cervical vessels; the level of SCC in the blood serum - $2.3 \mathrm{IU}$.

The data of the MRI of the pelvic and abdominal cavity were as follows: the cervix of an expressed granular heterogeneous nature; the thickness having unit anechoic fluid inclusions of up to $5 \mathrm{~mm}$; the internal os extended to $5 \mathrm{~mm}$; the cervical canal up to its middle third extended up to $6 \mathrm{~mm}$; the contours of the anterior and posterior lips - rough, clear, having hyperechoic linear strokes without acoustic effects; the blood flow in the thickness of the uterus of the mixed type; the ovaries of normal size, without pathology.

The set of the deviations evidenced the presence of invasive cervical cancer in stage T1a2NoMo which allowed conserving surgery. 
After preoperative preparations on 25.03.2011 the operation in the amount of radical abdominal trachelectomy was carried out (the access - lower midline laparotomy). The first step was performing iliac pelvic lymphadenectomy. The express cyto- and histological study gave no data of metastatic lesions of the lymph nodes. The examination revealed no distant metastases. The uterus was dense, normal in size. The fallopian tubes and the ovaries had no visual changes. The second stage included performing mobilization of the uterus, amputation of the cervix at the level of the upper third of the vagina and at the level of the internal os of the uterus. Sampling of biopsy specimens for cytoand histological examination was performed on the resection line. The conclusion: no tumour cells found. Next, a continuous anastomosis was applied between the uterus and vagina and a mesh implant in the form of a stocking having the width of $5 \mathrm{~cm}$ made of a superelasticTiNi thread was imposed around the anastomosis and fixed in the projection onto 3, 6, 9, 12 o'clock of the contingent dial.

The planned postoperative histological examination of the material №5453$99 / 12$ confirmed the presence of areas of squamous cell carcinoma with no keratinization with perifocal dense lymphoid infiltration. Along the resection line there was no tumour. Cancerous emboli were not detected.

The sutures were removed on the day 7-8 (the healing of the surgical wound per primam).

At the moment the case is followed up (once every 3 months) in the department of gynaecological oncology at the Federal State Funded Institution "Institute of Oncology". The follow-up examination after 12 months revealed no data of the tumour process progression. The speculum examination data: the vaginal mucosa of pale pink colour, visually not changed; the anastomosis line smooth, with clear boundaries, visually not changed; the uterus and the appendages of normal size, mobile, painless; the discharge - whites, lean. The cytology examination of the anastomosis line smears identified cells of stratified squamous epithelium without pathological changes.

According to the ultrasonography and MRI of the pelvic organs, the anastomosis zone had no pathological impurities. 


\section{Results and discussion}

Superelastic mesh implants of TiNi are widely used in current medical practice, including treatment of cancer patients. Advances in technologies of manufacturing thin threads of superelasticTiNi allow producing mesh structures whose elasticity is comparable to traditional sutures and dressings. Additionally, implants of TiNi treads have record biocompatibility and implantability, which removes the question of their subsequent disposal as required while using traditional materials [19].

The feasibility of using a mesh implant of superelasticTiNi is due to its biomechanical properties: it is not absorbed, but gets fused with the surrounding tissue and provides resistance of tissue to excessive strain. With regard to the issue of strengthening the utero-vaginal anastomosis, the implant takes over the function of the basic "closing apparatus" of the uterine and provides functional support and stability of the utero-vaginal anastomosis to the effects of intrauterine and intraperitoneal pressure. Having a developed surface, the mesh carries the main load and evenly distributes it over the area of the anastomosis eliminating the possibility of local effort concentration and associated fractures or pressure ulcers and fistulas.

The important point is the ability of the superelasticTiNi mesh to follow the shape of any surface without pre-deformation, which in relation to the problem makes it possible to lock the utero-vaginal anastomosis directly in the operation, providing the necessary "closing" effect. The mesh of TiNi threads with a superelasticity effect is involved in all deformation processes of the surrounding tissue because it can repeatedly return to its original state providing flexibility and robust durability of the formed anastomosis, which is important in view of a planned pregnancy in such patients. What is more, the mesh implant does not interfere with the process of coalescence between the contiguous uterus and vesicovaginal fascia.

The selected thread thickness in the range of $0.08-0.1 \mathrm{~mm}$ is due, on the one hand, to the technological capabilities of manufacturing thin threads available today, and on the other hand, to the need to ensure sufficient elasticity of the mesh structure. At the diameter of the thread of more than $0.1 \mathrm{~mm}$ the implant elasticity is considered insufficient. Development of the technology of producing TiNi threads allows predicting transition to even thinner threads $[6,19]$. 
The dimensions of the mesh cell in the range of $3-5 \mathrm{~mm}$ are due, on the one hand, to the ability to weave threads having ultimate flexibility, and on the other hand, to the possibility of preventing the prolapse of tissue into the lumen of the mesh. The width of the mesh implant within $4.5 \mathrm{~cm}$ corresponds to the anatomy of the defect providing a tissue covering on both sides of the anastomosis. Weaving the implant in the form of a stocking provides absence of traumatic ends of protruding metal wires and, moreover, the possibility of adapting the width of the stocking to the defect configuration. The length of the mesh implant in the range of 5-7 cm corresponds to the anatomy of the defect, which allows making at least one complete wrap around the anastomosis with a slight overlap. Imposing the mesh structure for tightening the anastomosis zone provides a transfer of pressure from the stitches onto the implant directly during the operation, which largely eliminates the likelihood of adverse effects. Fixation of the implant by surface stitches is not connected with occurrences of traumatic punctures and creates minimal effort, since it only immobilizes the implant for the nearest point preventing it from slipping.

In addition, an important point of managing this group of patients is the case follow-up within the postoperative period with the assessment of anatomical and functional viability of the anastomosis. In this regard, the use of the superelasticTiNi mesh is quite justified, since it does not create any obstacles for ultrasound monitoring during different periods of the observation. Furthermore, in view of the radio-opacity of the material, it is possible to conduct observational radiography to assess the status of the anastomosis.

\section{Conclusion}

Thus, at the present time conserving therapy in the amount of radical abdominal trachelectomy takes a worthy place among surgical procedures performed in modern gynaecological oncology. A promising area of research is evaluation of cancer effectiveness and analysis of long-term results after radical trachelectomy of various modifications, as well as development of rehabilitation actions, including psychological, physical and medical aspects. Studies on evaluation of fertility features, pregnancy, perinatal outcomes and patients' quality of life after organ-preserving treatment are of great interest. 


\section{References}

[1] E.L. Choinzonov, L.F. Pisareva, N.V. Cherduntseva, A.P. Boyarkina, I.N. Odintsova, N.A. Martynova, Cancer incidence in Siberia and the Russian Far East, Cancer care services, Bulletin of Siberian Branch of the Russian Academy of Medical Sciences [in Russian].2 (2004) 41-47.

[2] E.L. Choinzonov, L.F. Pisareva, L.D. Zhuikova, Cancer incidence in Tomsk region for the period from 2004 to 2009, Assessment of diagnosis quality, Siberian Journal of Oncoplogy [in Russian]. 3 (2011) 29-34.

[3] L.V. Adamyan, K.I. Zhordania, S.M. Beloborodov, Reproductive function in cancer patients, How to maintain ability to have children, Oncology Problems. 503 (2004) 279-292.

[4] I. Palaia, M. Pernice, M. Graziano, Neoadjuvant chemotherapy plus radical surgery in locally advanced cervical cancer during pregnancy: a case report, Am. J. Obstet. Gynecol. 197 (2007) 5-6.

[5] V.A. Antipov, E.G. Novikova, O.S. Balakhontseva, A.S. Shevchuk, Functional and anatomic rehabilitation after radical abdominal trachelectomy, Problems of reproduction, 1 (2010) 103107.

[6] A.L. Chernyshova, A.Yu. Lyapunov, L.A. Kolomiets, V.I. Chernov, I.G. Sinilkin, Detection of sentinel lymph nodes in surgical treatment of cervical cancer, Siberian Journal of Oncology [in Russian]. 51 (2012) 28-33.

[7] L. Ungar, L. Palfalvi, R. Hogg, Abdominal radical trachelectomy: a fertility-preserving option for women with early cervical cancer, Br.J. Gynaecol. 112 (2005) 366-369.

[8] D. Dargent, Radical trachelectomy: an operation that preserves the fertility of young women with invasive cervicalcancer, Bull. Acad. Natl. Med. 185 (2001) 1295-304.

[9] L. Ungar, Update on radical abdominal trachelectomy, Abstracts of International Video Workshop on radical surgery in gynecological oncology, Prague, 2008.

[10] Dargent D, Martin X, Sacchetoni A. Laparoscopic vaginal radical trachelectomy: a treatment to preserve the fertility of cervical carcinoma patients // Cancer. 2000. 88:1877-1882.

[11] V.E. Gunther, Medical Materials and Shape Memory Implants [in Russian], MIC, Tomsk, 2013.

[12] V.A. Antipov, E.G. Novikova, O.S. Balakhontseva, A.S. Shevchuk, Radical abdominal trachelectomy: technical aspects, Oncology Problems. 56 (2010) 36-42.

[13] D. Dargent, Using radical trachelectomy to preserve fertility in early invasive cervical cancer, Contemporary OB/GYN, 2000, 23-49.

[14] E.G. Novikova, V.A. Antipov, E.A. Ronina, O.S. Balakhontseva, Radical abdominal trachelectomy, Russian Oncology Journal [in Russian]. 1 (2010) 8-14.

[15] A.L. Chernyshova, L.A. Kolomiets, S.E. Krasilnikov, Organ-preserving surgery for invasive cervical cancer, Siberian Journal of Oncology [in Russian]. 51 (2011) 72-78.

[16] A. Karam, N. Feldman, C.H. Holschneider, Neoadjuvant cisplatin and radical cesarean hysterectomy for cervical cancer in pregnancy, Nat. Clin. Pract. Oncol. 4 (2007) 375-380.

[17] S. Alouini, K. Rida, P. Mathevet, Cervical cancer complicating pregnancy: implications of laparoscopic lymp hadenectomy, Gynecol Oncol. 108 (2008) 472-477. 
[18] F. Amant, K. Van Calsteren, M. Halaska, Gynecologic Cancers in Pregnancy: Guidelines of an International Consensus Meeting, Int. J. Gynecol. Cancer. 19 (2009) 1-13.

[19] D. Dargent, J.L. Burn, M. Roy, I. Remi, Pregnancies following radical trachelectomy for invasive cervical cancer, Gynecol. Oncol. 52 (1994) 105.

[20] C.M. Salafia, V.K. Minior, J.A. Lopez-Zeno, S.S. Whittington, J.C. Pezzullo, A.M. Vintzileos, Relationship between placental histo, Int.J. Med. Sci. 20 (2010) 67-71. 